\title{
Preface
}

\section{Material standards and reference materials for disease biomarkers: Needs assessment and development}

\author{
Peter E. Barker \\ NIST Division of Biochemical Sciences, Chemical Sciences and Technology Laboratory, National Institute of \\ Standards and Technology, Gaithersburg, MD, USA
}

The concept of assay controls is so familiar, most clinical and research scientists give it no second thought. Controls account for assay variability beyond the dependent variable under study. Controls are widely used in every experiment and clinical assay daily, and are part of standard laboratory protocol for each individual clinical laboratory and research group.

Reference materials are a means to harmonize the results of measurements among multiple laboratories. These can be informally implemented by collaborating laboratories, or developed more formally by professional organizations and, in some cases, by state and federal agencies.

For clinical chemistry and biomedical applications, reference materials (RMs) or certified reference materials (CRMs) may take the form of neat materials of assessed purity, or solutions of analytes. These are typically used for calibration purposes. Many are in the form of matrix materials with assigned values of various analytes of interest.

Matrix materials are commonly used to validate that a measurement process provides results that are fit for a defined purpose, or to verify that a measurement process is performing as required. A Standard Reference Material ${ }^{\circledR}(\mathrm{SRM})$ is a CRM issued by NIST that meets additional criteria as established by the United States National Institute of Standards and Technology (NIST) [1]. As SRMs are precious commodities, they typically are not intended for routine clinical or ex- perimental applications as controls. Intended use descriptions often accompany supporting documentation of such standards.

NIST's role in early cancer biomarker evaluation is outlined [2]. As part of continued NIST commitment to healthcare [3], recent NIST workshops have addressed standards and technology in areas such as nanotechnology and early cancer detection [4], HER2 testing of breast cancer [5], gene expression [6] and serum proteomics for early cancer detection [7].

Standards arise in response to clinical needs. In some cases, professional groups or laboratory associations initiate the process. Working in partnership with the standards user communities, NIST serves the national measurements community and has the specific federally mandated responsibility to meet high-priority reference material needs.

Various phases of this needs assessment and reference development process in the molecular diagnostics and cancer biomarkers community, and at NIST, are illustrated by the papers in this issue of Disease Markers. Michaud and Winn-Deen address the molecular diagnostic standards needs and issues in the US. Food and Drug Agency (FDA) and in the private sector biotechnology community, respectively. Amos details some pressing recent issues in measurement of autoantibodies that will be discussed in an upcoming USMS Autoantibody Workshop at NIST on February 21-22, 2006. Finally, two papers report on standards devel- 
opment for HER2 testing in the European Community (Rhodes), and molecular diagnostics standards at NIST (O'Connell, Atha and Jakupciak).

In an era of increasingly high-dimensional data sets for clinical work such as gene expression microarrays [6] and serum proteomics [7], standards along the entire measurement certification pathway are critically important to promote development and commercialization. If the promise of biotechnology is to be realized in this exciting new era, careful forethought is appropriate as to identify which diagnostic standards are needed, how these can be prioritized, implemented and supported, and how these can leverage accurate, costeffective, high-throughput technology to the benefit of the healthcare consumer.

\section{References}

[1] W. May, R. Parris, C. Beck, J. Fassett, R. Greenberg, F. Guenther et al., Standard Reference Material $s^{\circledR}$ Definitions of terms and modes used at NIST for value-assignment of reference materials for chemical measurements, NIST Special Publica- tion 2001, 260-136. Washington, DC: US Department of Commerce.

[2] P.E. Barker, Cancer biomarker validation, standards and process: roles for the National Institute of Standards and Technology, Ann. N. Y. Acad. Sci. (Epigenetics and Cancer Detection issue, edited by M. Verma). (review) 983 (2002), 142-150.

[3] D.L. Duewer, J.B. Thomas, M.C. Kline, W.A. MacCrehan, R. Schaffer, K.E. Sharpless, W.E. May and J.A. Crowell, NIST/NCI micronutrients measurement quality assurance program: measurement repeatabilities and reproducibilities for fatsoluble vitamin-related compounds in human sera, Anal Chem 69 (1997) ,1406-1413.

[4] S. Zullo, P. Srinivas, S. Srivastava, J.P. Looney and P.E. Barker, Nanotechnology: roles in early detection of cancer, Disease Markers 18 (2002), 153-158.

[5] E. Hammond, P.E. Barker, S. Taube and S. Gutman, Standard reference material for Her2 testing: report of a National Institute of Standards and Technology-sponsored Consensus Workshop, Appl. Immunohistochem. Mol. Morphol. 11 (2003), 103-106.

[6] M. Cronin, K. Ghosh, F. Sistare, J. Quackenbush, V. Vilker and C. O'Connell, Universal RNA reference materials for gene expression, Clinical Chemistry 50 (2004), 1464-1471.

[7] P.E. Barker, P.D. Wagner, S.E. Stein, D.M. Bunk, S. Srivastava and G.S. Omenn, 2006. Standards for Plasma and Serum Proteomics in Early Cancer Detection: A Needs Assessment Report from the NIST-NCI SMART Workshop, August 18-19, 2005 (submitted). 